\title{
Optimum Wavelet Selection for Orthogonal Wavelet Division Multiplex (OWDM) Based Broadcasting System
}

\author{
Mohammed Tarique \\ Department of Electrical Engineering \\ Ajman University of Science and Technology \\ Fujairah, United Arab Emirates \\ m.tarique@ajman.ac.ae
}

\begin{abstract}
In the recent years Orthogonal Wavelet Division Multiplexing (OWDM) has been considered as an alternative of Orthogonal Frequency Division Multiplexing (OFDM). OWDM has lower computational complexity and higher flexibility compared to its OFDM counterpart. Wavelet is the most important element of an OWDM based system. Wavelets have been a much investigated topic in digital image processing for a long time. Recently they are being used in efficient communication system design. In this work we use wavelets in Digital Video Broadcasting-Terrestrial (DVB-T) system. Originally DVB-T system was designed based on OFDM. In this paper we use OWDM instead. We apply a number of wavelets namely Haar, Daubechies, Biorthogonal, Coiflets, and Symlets in OWDM design. The simulation results show that the Haar function outperforms other wavelets and hence this function should be used in OWDM based DVB-T broadcasting system.
\end{abstract}

Keywords: DVB, OFDM, OWDM, wavelets, AWGN, Haar, Daubechies, Coiflets, Bi-orthogonal, Symlets

\section{Introduction}

Digital Video Broadcasting (DVB) was introduced by DVB group, which is a consortium of over 200 companies and regulatory bodies across Europe [1]. The main motivation was to convert analog television system into digital television system. The origin of DVB system is dated back to 1969 [2] when Chang demonstrated a novel approach to transmit signal at a very high rate (close to Shannon's rate). Initially, DVB was introduced for stationary signal reception by using rooftop antenna. Extensive researches demonstrated that DVB signal could also be received by mobile devices mounted on high speed vehicles [3]. The United Kingdom (UK) was one of the first European countries to launch terrestrial digital television services based on the DVB system followed by other European countries. Till date the DVB system has been considered as one of the most popular technology in digital video broadcasting around the World. Many commercial versions of DVB systems have been introduced. Among them DVB-S, DVB-C, and DVBThave become very popular for satellite, cable, and terrestrial networks respectively. Detail descriptions of these technologies can be found in [4-5]. Since this work is based on DVB-T we present a summary of DVB-T here for completeness of our work. 
Mohammed Tarique; Optimum Wavelet Selection for Orthogonal Wavelet Division Multiplex (OWDM) Based Broadcasting System, Transactions on Networks and Communications, Volume 4 No. 4, August (2016); pp: 1-12

DVB-T system operates within the existing Very High Frequency (VHF) and Ultra High Frequency (UHF) bands. Two modes of operation have been defined for the DVB-T system namely " $2 \mathrm{~K}$ mode" and " $8 \mathrm{~K}$ mode". The signal transmission of the DVB-T system is based on Moving Picture Experts Group-Two (MPEG-2). The DVB-T signal undergoes channel coding and modulation before it is transmitted. It allows different levels of Quadrature Amplitude Modulation (QAM) and uses different code rates. The other signal processing steps done in the transmitter include multiplexing, multiplex adaptation, outer coding, outer interleaving, inner coding, inner interleaving, mapping, frame adaptation, OFDM, and guard interval insertion as shown in Figure1. The digital signal generated by the above mentioned signal processing steps is then converted into an analog signal by using a Digital-to-Analog (D/A). Most of the signal processing steps shown in Figure1 is done in a digital signal processor. The performance of DVB system highly depends on the modulation, which is OFDM.

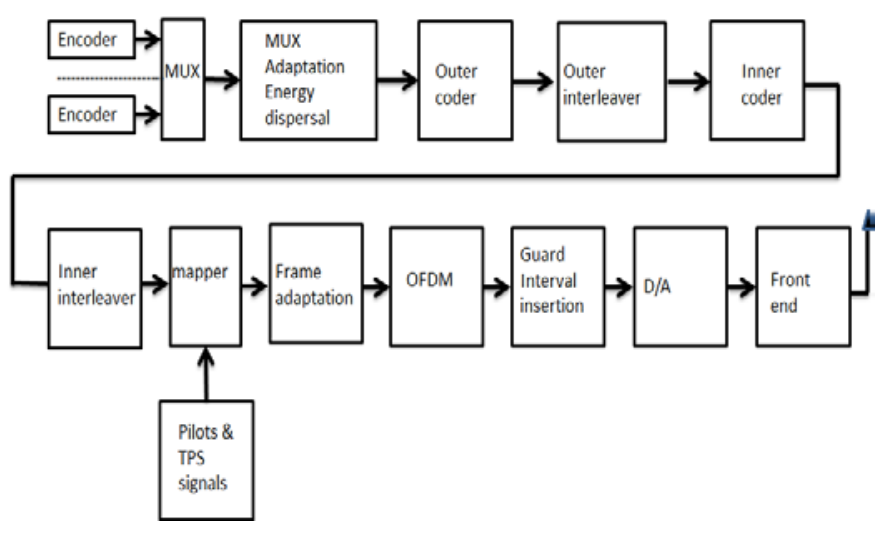

Figure. 1 Signal processing steps in the DVB-T transmitter

Orthogonal Frequency Division Multiplexing (OFDM) is a multi-carrier modulation (MCM) scheme [6-7]. The main principle of MCM is to divide the input bit stream into several parallel bit streams and then these bit streams are used to modulate several sub-carriers as shown in Figure 2. In the receiver side band-pass filters are used to separate the spectrum of individual subcarriers. OFDM is a special form of spectrally efficient MCM technique, which employs densely spaced orthogonal sub-carriers and overlapping spectrums. Hence, it is considered as a spectrum efficient modulation scheme. Depending on the channel coherence bandwidth OFDM reduces or completely eliminates the effects of Inter Symbol Interference (ISI). The Inverse Discrete Fourier Transform (IDFT) is used to implement the orthogonal signals in OFDM transmitter. The Discrete Fourier Transform (DFT) counterpart is used in OFDM receiver.

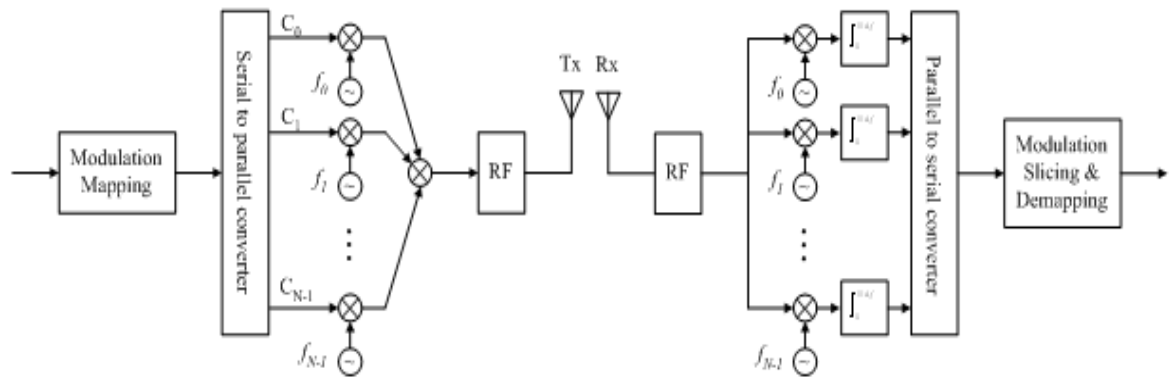

Figure. 2 The OFDM System 
OFDM has numerous advantages over other modulation schemes. The main advantage of OFDM is that it can minimize Inter Symbol Interference (ISI) originated from the multipath fading channel [8]. Hence, it can support a very high data rate. There are some other advantages too. OFDM can cope with the multipath fading with less computational complexity. Because it uses computationally efficient Fast Fourier Transform (FFT) and Inverse Fast Fourier Transform (IFFT) algorithms. OFDM is also considered suitable for coherent demodulation. Additionally, OFDM can ensure the required Quality of Service (QoS) to the end users depending on the bandwidth. It is also considered suitable for diversity techniques (i.e., time diversity and frequency diversity).

OFDM has some disadvantages too. It requires a strong synchronization between transmitter and receiver. It is very much sensitive to phase noise and frequency offset. It is not power efficient because the Fast Fourier Transform (FFT) algorithm and Forward Error Correction (FEC) have to remain active all the time. It cannot take the advantages of the diversity gain when a few sub-carriers are allotted to each user. In order to avoid amplitude distortion OFDM requires a high peak to average ration RF power amplifier.

Alternatives of OFDM have been proposed in the literatures. Orthogonal Wavelet Division Multiplexing (OWDM) is one of them. In order to investigate the effectiveness of OWDM over OFDM we choose practical digital broadcasting system called Digital Video Broadcasting-Terrestrial (DVB-T). Originally, DVBT system was designed based on OFDM. In this work we use OWDM instead. The rest of the paper is organized as follows. The basic principles of OWDM have been presented in section II. Some related works have been presented in section 2 . The architecture of OWDM has been presented in section 3 . The wavelet families and their properties have been presented in section 4 . The simulation model and results have been presented in section 5 . The paper has been concluded with section 6 .

\section{Related Works}

One of the early works on wavelet-based DVB-T system can be found in [14]. In this work a comparison of Fourier-based OFDM system and wavelet-based OWDM system has been presented. It is shown that the OWDM based DVB-T system out-performs the Fourier-based OFDM in terms of bit error rate for a given signal-to-noise ratio. To implement OWDM system the authors choose Daubechies family only. The authors consider two channel conditions namely AWGN and Rayleigh fading channel. It is shown that the OWDM based DVB-T out-performs Fourier-based DVB-T under the both channel conditions.

In another early work [11] the author has shown that the wavelet-based OWDM is more computationally efficient compared to Fourier-based OFDM system. It is shown that OWDM is also more flexible and noise resilient compared to Fourier-based OFDM system. Wavelet families including Symlet, Coiflet, Daubechies, and Haar have been investigated in the same work. It is shown that the Haar wavelet is the best choice for DVB-T system. The major limitation of the work is that the author only considers AWGN channel model.

A comprehensive analysis of the performance of DWT-OFDM system has been presented in [15]. The authors have compared these performances against the same of FFT-OFDM system under AWGN channel condition. They argue that DWT-OFDM does not require cyclic prefix. Hence, it can be considered more spectrally efficient compared to FFT-OFDM. The authors also investigate other performance parameters including peak-to-average ratio (PARP). It is shown that the value of PAPR is also much less in DWT-OFDM than that in conventional FFT-OFDM. The authors conclude that the DWT-OFDM should be considered 
Mohammed Tarique; Optimum Wavelet Selection for Orthogonal Wavelet Division Multiplex (OWDM) Based Broadcasting System, Transactions on Networks and Communications, Volume 4 No. 4, August (2016); pp: 1-12

always a better candidate for DVB-T system than FFT-OFDM. The authors also defend that DWT-OFDM is more power efficient and hence it is a good candidate for DVB-H. Because DVB-H has been designed for handheld devices, which have limited battery capacity.

It has been shown in [16] that the wavelets can be favorably applied in all aspects of digital wireless communication system including transceiver design, de-noising, channel coding, and channel modelling. The authors conclude that DWT-OFDM has less bit error rate and lower PAPR compared to FFT-OFDM. Additionally, cyclic prefix (CP) can be avoided in DWT-OFDM that makes it more bandwidth efficient compared to FFT-OFDM. The authors argue that approximately $20 \%$ of the bandwidth of FFT-OFDM can be saved in DWT-OFDM for not using CP. An additional bandwidth saving of $8 \%$ can also be achieved by not using pilot tones.

Better Wavelet Packet Tree (BWPT) structure has been proposed in [17] for wavelet-based OFDM (WOFDM) system. A brute force search algorithm has been used to obtain BWPT structure. The authors suggest Daubechies (dB) wavelets for implementing WOFDM. They argue that Daubechies based WOFDM reduces PAPR without any additional complexity because of BWPT structure.

A novel dual-tree complex wavelet transform (DT-CWT) scheme has been proposed for OFDM in [18]. The authors have shown that the proposed scheme shows better performance in terms of BER and PAPR than the conventional OFDM. The other advantage of DT-CWT is that it has less computational complexity and it eliminates CP due to the good orthogonality and time frequency localization property.

In a similar work [19] the performance of bit error rate (BER) of Discrete Fourier Transform (DFT) based OFDM has been compared with that of wavelet based OFDM system. The power spectral density (PSD) of these two different OFDM systems have also been compared in the same work. The authors also compare the performances of the both systems by using two channel estimation techniques namely Least Square (LS) and Linear Minimum Mean Square Error (LMMSE). The simulation results show that wavelet based OFDM performs better than DFT based OFDM system. Moreover, the PSD of the wavelet based OFDM is more compact compared to its DFT based counterpart.

\section{The OWDM System}

Now-a-days, Orthogonal Wavelet Division Multiplexing (OWDM) has been considered as an alternative solution of OFDM. In order to design OWDM based DVB-T system we replace the OFDM block shown in Figure1 by an OWDM block. OWDM system uses wavelet functions. The wavelets have been used for a long time in the field of digital image processing. Recently, they have been used in communication system design. The wavelet has many advantages over Fourier transform. The main advantage is that the wavelet can provide accurate information about the fast fluctuations of the signals in the time domain. It maps a time functions into two functions namely scale, a and translation, $b$. The continuous wavelet transform (CWT) of a signal $x(t)$ is defined as

$$
W(a, b)=\int_{-\infty}^{\infty} f(t) \psi_{a b}(t) d t
$$

, where $W(a, b)$ is the wavelet transform and $\psi_{a b}(t)$ is called the mother wavelet, which is defined as

$$
\psi_{a b}(t)=\frac{1}{\sqrt{a}} \psi\left(\frac{t-b}{a}\right)
$$


A scaled version of the function $\psi(t)$ with a scale factor of $a$ is defined as $\psi(t / a)$. There are a number of mother wavelets available. Since in this paper we focus on digital system we use Discrete Wavelet Transform (DWT) instead. It has been shown in [9] that it is possible to reconstruct DWT from CWT by using a bank of high pass filter and low pass filter as shown in Figure 3. In this figure three sub-bands decomposition has been depicted. In real life usually more sub-bands decomposition is performed. The filters generate more data by convolving the input signal with filter impulse response. Hence, down sampling operation is done during decomposition by discarding the alternate samples. The synthesis process of the DWT decomposed signal is also depicted Figure3. In order to compensate the down sampling operation done during decomposition up sampling is done in wavelet synthesis by inserting zeroes in the data. The main property of the wavelet transform is that it contains both frequency information and time information. Hence, it can convey a high data rate within each sub-band.

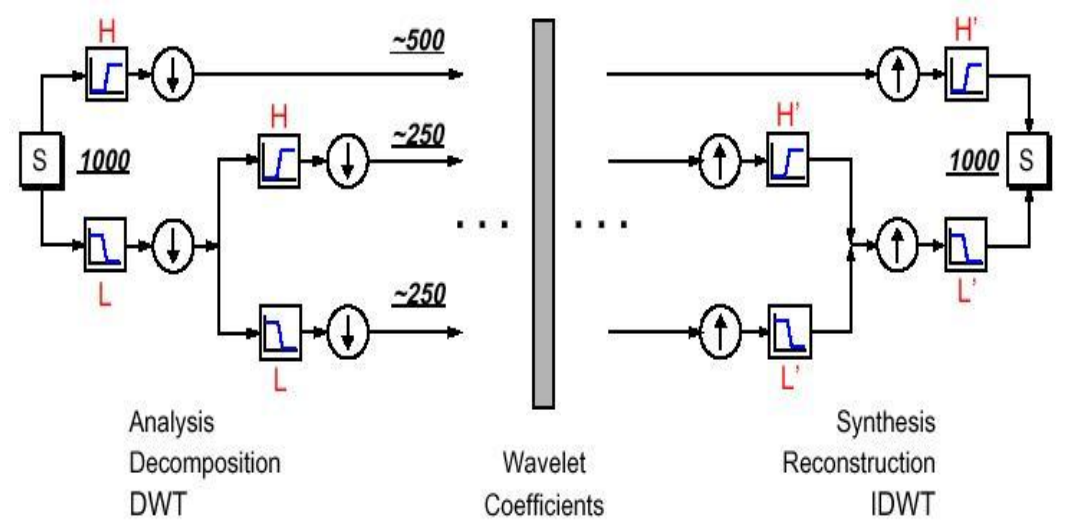

Figure 3 The Wavelet decomposition and synthesis

Contrary to the IFFT operation done in OFDM system based transmitter Inverse Discrete Wavelet Transform (IDWT) operation is done in OWDM system based transmitter. The output of the IDWT can be represented as [13]:

$$
s(k)=\sum_{m=0}^{\infty} \sum_{n=0}^{\infty} S_{m}{ }^{n} 2^{m / 2} \psi\left(2_{k}{ }^{m}-n\right)
$$

where $\left\{S_{m}{ }^{n}\right\}$ are the wavelet coefficients and $\psi(t)$ is the wavelet function that is compressed $m$ times and shifted $\mathrm{n}$ time for each subcarrier. In the receiver the inverse operation (i.e., Discrete wavelet Transform is done). The output of discrete wavelet transform (DWT) is represented as:

$$
S_{m}{ }^{n}=\sum_{k=0}^{N-1} s(k) 2^{m / 2} \psi\left(2_{k}{ }^{m}-n\right)
$$

The output signal $\left\{S_{m}{ }^{n}\right\}$ is then decoded to data to QAM modulator.

\section{The Wavelet Families}

Numerous mother wavelet and their families [9] have been proposed and used in different fields of signal processing including image processing. These wavelets vary in terms of symmetry, regularities, vanishing moments, and the length. In this work we focus on two important criteria namely scaling function and orthogonality (or bi-orthogonality). In each wavelet families there are child wavelets that are 
Mohammed Tarique; Optimum Wavelet Selection for Orthogonal Wavelet Division Multiplex (OWDM) Based Broadcasting System, Transactions on Networks and Communications, Volume 4 No. 4, August (2016); pp: 1-12 distinguished by coefficients and number of iteration. Since we are considering wavelet as an alternative of OFDM, we consider the orthogonality and bi-orthogonality property as our prime concerns.

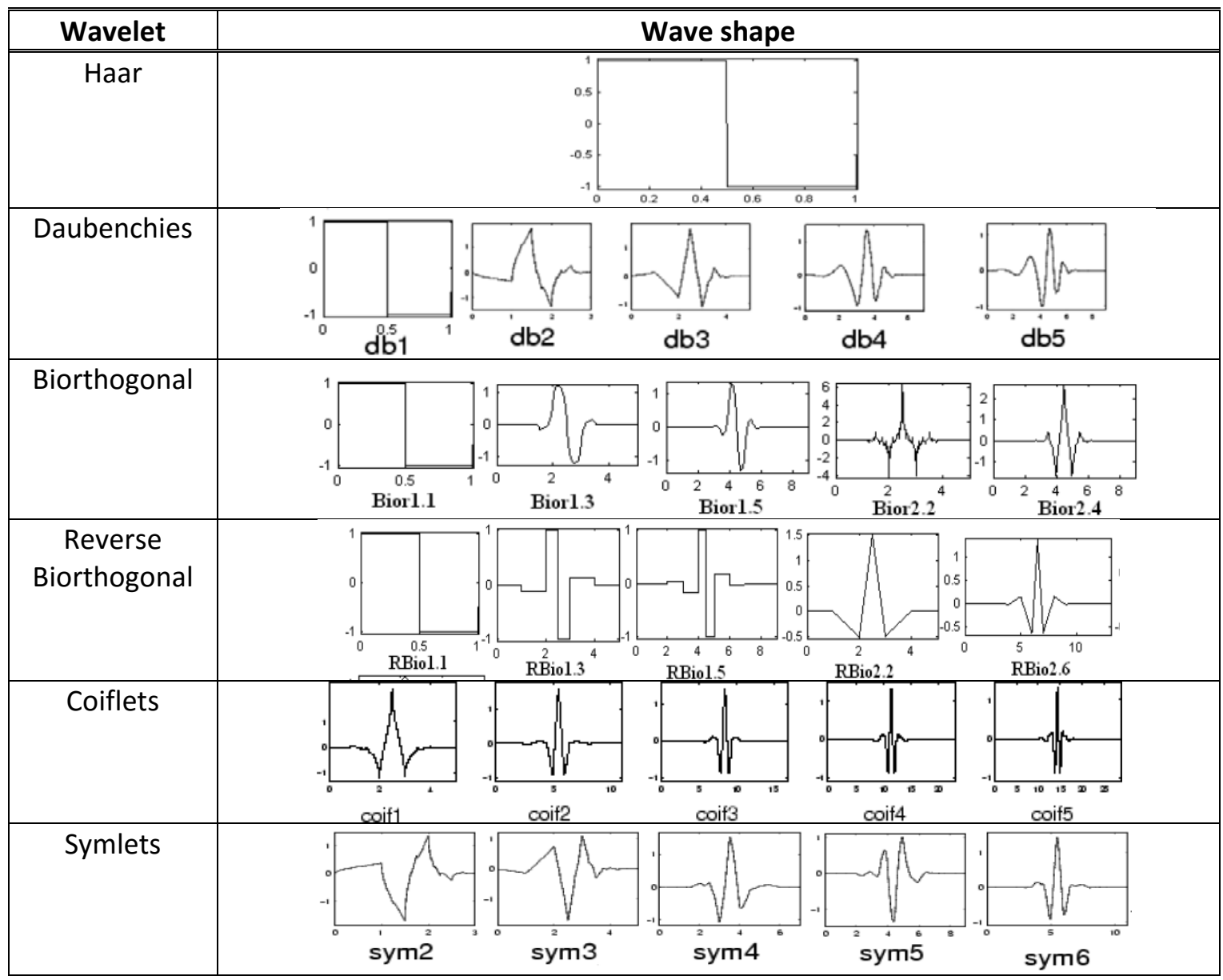

Figure.4 The wavelets and their families [12]

Among the wavelets families available in the literature we consider only Haar, Daubechies, Biorthogonal, Reverse Biorthogonal, Coiflets, and Symlets wavelet families as the candidates for our OWDM simulation. These wavelet families have various degree of orthogonality. The Haar wavelet function was proposed by Afred Haar in 1909. Among all the wavelets considered in this work the Haar wavelet is the simplest one and memory efficient. It is not continuous as shown in Figure 4. It has only one vanishing moment. The Haar function is considered only suitable for the signals with sharp transition.

The Daubechies wavelet is named after the Belgian physicist and mathematician Ingrid Daubechies. The Daubechies wavelets are orthogonal and have maximal number of vanishing moments. Among all the available Daubechies wavelets mostly used family are $\mathrm{db} 2-\mathrm{db} 20$. The index indicates the vanishing moments. For example, $\mathrm{db} 2$ has two vanishing moments, and db3 has three vanishing moments etc. The $\mathrm{db} 1$ has one vanishing moment and referred to as the Haar wavelet. Another peculiar characteristic of the Daubechies wavelets is that they are not symmetric 
Another popular wavelet family is Biorthogonal wavelets. They find many applications in signal processing due to their linear phase property. The linear phase property is achieved by maintaining symmetry in the filter coefficients. The Biorthogonal wavelets provide more degree of freedom compared to their orthogonal wavelets. The Biorthogonal wavelets are compactly supported symmetric. The Reverse Biorthogonal wavelets family is obtained from the Biorthogonal wavelet families. Both Biorthogonal and Reverse Biorthogonal wavelet families are compactly supported.

Table I: The Properties of Wavelet Families

\begin{tabular}{|c|c|c|c|c|c|c|}
\hline Property & Haar & Daubechies & Symlet & Coiflet & Biorthogonal & $\begin{array}{c}\text { Reverse } \\
\text { Biorthogonal }\end{array}$ \\
\hline Symmetry & $\mathrm{X}$ & $\mathrm{X}$ & $\mathrm{X}$ & & $\mathrm{X}$ & $\mathrm{X}$ \\
\hline Asymmetry & & $\mathrm{X}$ & $\mathrm{X}$ & $\mathrm{X}$ & $\mathrm{X}$ & \\
\hline $\begin{array}{c}\text { Near } \\
\text { symmetry }\end{array}$ & & $\mathrm{X}$ & $\mathrm{X}$ & $\mathrm{X}$ \\
\hline $\begin{array}{c}\text { Arbitrary } \\
\text { number of } \\
\text { vanishing } \\
\text { moments }\end{array}$ & $\mathrm{X}$ & $\mathrm{X}$ & $\mathrm{X}$ & & \\
\hline $\begin{array}{c}\text { Compactly } \\
\text { Orthogonal }\end{array}$ & $\mathrm{X}$ & & & $\mathrm{X}$ & $\mathrm{X}$ \\
\hline $\begin{array}{c}\text { Compactly } \\
\text { Biorthogonal }\end{array}$ & & & & & \\
\hline
\end{tabular}

The Coiflet wavelets family was introduced by Daubechies and was named in the honor of Ronald Coifman. The peculiar characteristic of the Coiflet wavelets is that they are more symmetrical than the Daubechies wavelet. The Symlet wavelets are compact, orthogonal, and continuous. These wavelets are less symmetric compared to the Daubechies wavelets. A comparison of the investigated wavelets is listed in Table 1.

\section{System Simulation and Results}

The transmitter and the receiver models presented in [10] have been used in this investigation. Since the number of carriers in DVB-T system is 1705 , we consider 1705 4-QAM symbols as the input data to the transmitter model. We use a bandwidth of about $7.61 \mathrm{MHz}$. The carrier frequency we choose is $90 \mathrm{MHz}$. Since the disadvantages of OFDM signal occur in continuous time domain, we convert the output carriers of IFFT into continuous time signal. This step of the signal processing is accomplished by using a pulse shaping filter and a low pass filter denoted by Digital-to-Analog (D/A) filter in the block diagram of Figure5(a). The pulse shaping filter converts the discrete time carrier output signals into continuous signal. In the next step a D/A filter, with a sharp bandwidth, is used. We choose a Butterworth filter for this purpose.

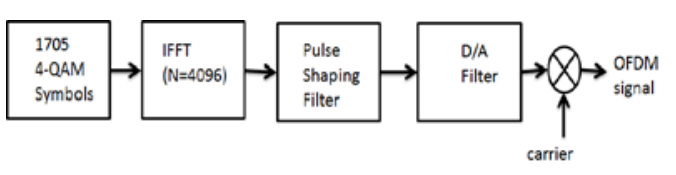

(a) Transmitter model

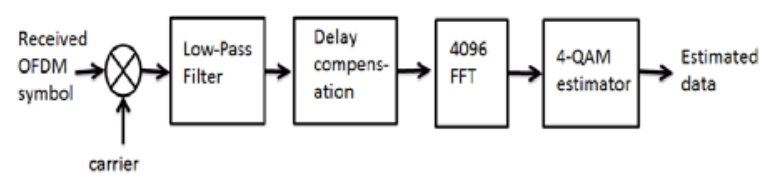

(b) Receiver model

Figure. 5 OFDM Transmitter and Receiver model 


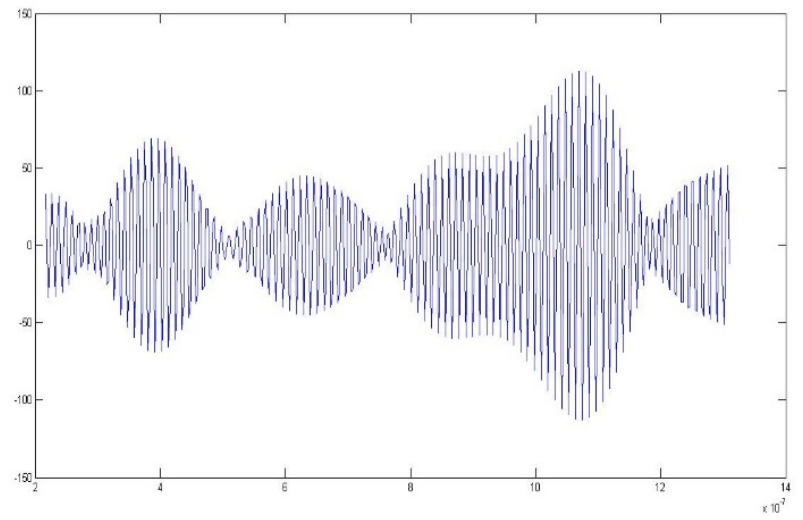

Figure. 6 The FFT based DVB-T Signal

The carrier modulation is performed at the last stage. The DVB-T generated by the transmitted model is shown in Figure 5(b). The generated DVB-T signal is depicted in Figure 6. It shows that that the DVB-T signal exhibits a large variation in the amplitude.

Since its introduction OFDM receiver design has been an active research area. Numerous researches have been carried out and innovations have been done in the OFDM receiver design. The OFDM receiver model used in this investigation is shown in Figure 5(b). The OFDM receiver operation performs the inverse operation that were done in the transmitter. First, coherent demodulation is done. The demodulated OFDM signal is then passed through the low-pass filter to recover the continuous version of the OFDM data. The delays produced by reconstruction and demodulation filters are compensated. The resultant signal is then sampled to convert continuous OFDM symbol into its discrete version. The resulted signal is then passed through FFT to do the inverse function of its counterpart (i.e., IFFT) done in the transmitter. Finally, the resultant data is estimated by using a 4-QAM estimator. The scatter plot for the DVB-T received signal under Additive White Gaussian Noise (AWGN) channel at Signal-to-Noise Ratio (SNR) of 5 dB is shown in Figure7. Compared to the scatterplot of a noise free 4-QAM constellation the received signal point are shown scatted far from the idea constellation points, which are $(-1,-1),(-1,1),(1,-1)$, and $(1,1)$.

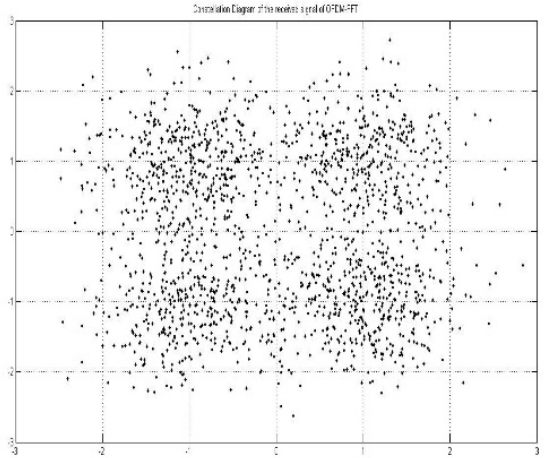

Figure. 7 The scatterplot for the received FFT based DVB-T signal at SNR of $5 \mathrm{~dB}$.

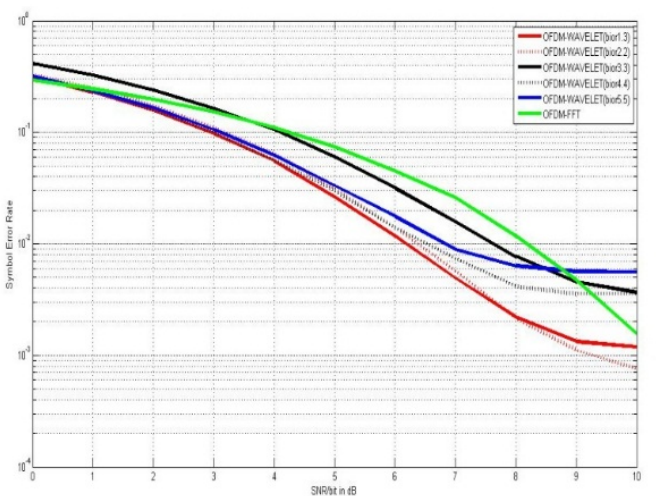

Figure. 8 The results of Biorthogonal families 
The main idea of this work is to investigate the performance of DVB-T system using different wavelets in the modulator under the same AWGN channel model. The advantages of the wavelet based DVB-T over FFT based DVB-T have been listed in [11]. The major advantages include increase in flexibility, increase in resilience, and decrease in computational complexity. Compared to the FFT based OFDM system the wavelet based OWDM allows more parameters to be varied. For example, in OWDM each subcarrier can have different coding and modulation. In OWDM more error correction can be applied to the affected sub-bands. Hence, OWDM is more resilient to frequency selective fading. The computational complexity in OWDM is less than that of the FFT based OFDM. The computational complexity in the FFT based OFDM is $N \log _{2} N$ whereas the same is only $N$ in OWDM system [11].

We construct another simulation model for the wavelets. The only difference is that we replace the IFFT block in the transmitter by an IDWT block. Similarly, we replace the FFT block in the receiver by a DWT block. Then, we use different wavelets to compute the IDWT and DWT. The simulation results of the DVBT system under different wavelet families are shown in Figure8, Figure9, Figure10, Figure11, Figure12 and Figure13.

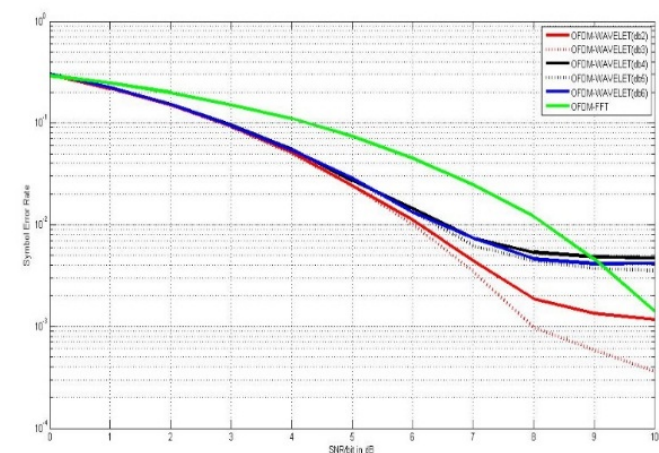

Figure. 9 The results of Daubechies families

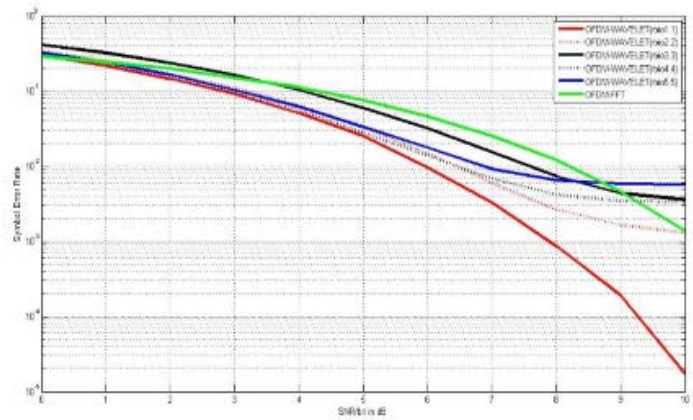

Figure 11 The results of Reverse Biorthogonal families

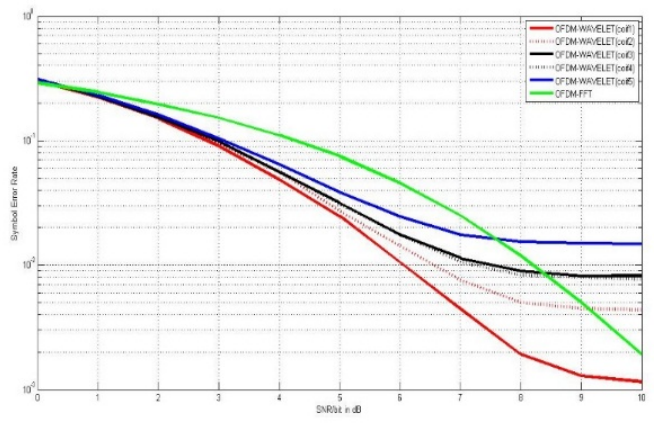

Figure 10 The results of Coiflet families

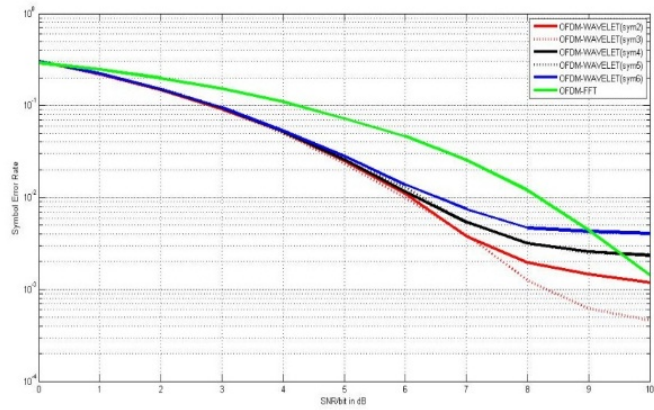

Figure 12 The results of Symlet families

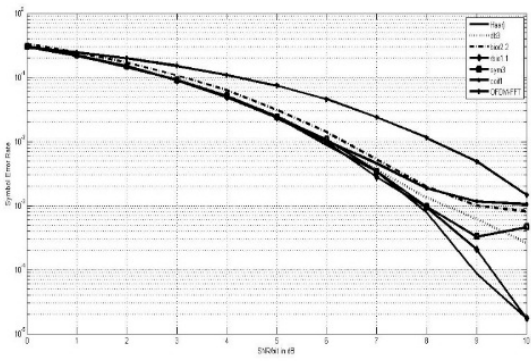

Figure 14 The comparison of the best wavelets 
Mohammed Tarique; Optimum Wavelet Selection for Orthogonal Wavelet Division Multiplex (OWDM) Based Broadcasting System, Transactions on Networks and Communications, Volume 4 No. 4, August (2016); pp: 1-12 The simulation result of the FFT based DVB-T has also been plotted in the same figure for fair comparison. The simulation results show that wavelet based DVB-T system outperforms (more or less) the FFT based DVB-T for all wavelet families. Among the wavelet and their families Bi-orthogonal 2.2, Daubechies 3, Coiflet 1, Reverse Bi-orthogonal 1.1, and Symlet 3 wavelets are the best candidates for the DVB-T system. In order to choose the best wavelet we compare the performances of the DVB-T systems for Bi-orthogonal 2.2, Daubechies 3, Coiflet 1, Reverse Bi-orthogonal 11, and Symlet 3 with that of much investigated Haar wavelet in Figure 13. The simulation results show that the Haar function is the optimum choice for the DVB-T system. The Biorthogonal is the least resilient wavelet family for DVB-T system followed by the Symlet and Coiflet. The performance of the Reverse Biorthogonal wavelet is almost similar to that of the Haar wavelet

In order to investigate the PAR we compare the signal generated by FFT based DVB-T transmitter as shown in Figure 6 to that of Haar wavelet based DVB-T transmitter as shown in Figure15. Comparing these two signals we conclude that the signal amplitude variation is less in wavelet based system. In order words the wavelet based OFDM system is more useful to solve the very well-known of OFDM system's high PAR ratio problem.

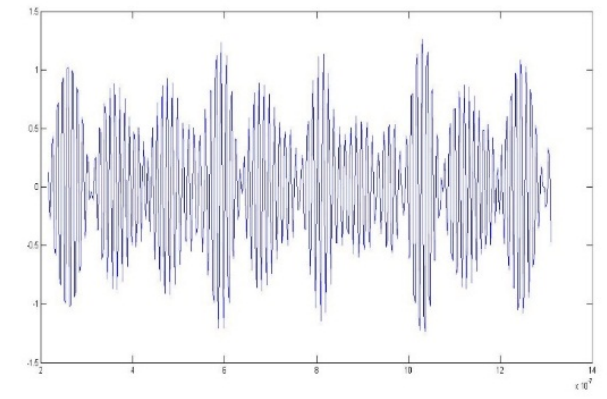

Figure 15 The transmitted signal for Haar based DVB-T system

The scatterplot of the received signal in Haar wavelet based DVB-T system is shown in Figure 15. Examining the scatterplot of Haar wavelet based DVB-T presented in Figure 15 and the same for the FFT based DVBT presented in Figure7, we can conclude that the received constellation points in wavelet based system are more close to the ideal constellation points. It means that the OWDM based DVB-T signal is more resilient to channel impairments compared to it OFDM counterpart under the AWGN channel condition.

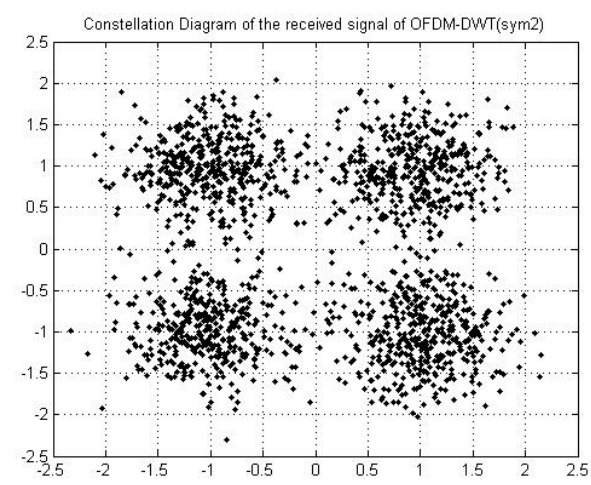

Figure 15 The scatterplot for the received Haar wavelet based DVB-T signal 


\section{Conclusion}

In this work the effects of different wavelets on the performance of the DVB-T system have been investigated. Although the DVB-T system originally designed based the Fast Fourier Transform (FFT), many researchers have discovered that the FFT based DVB-T system has some limitations including high computational complexity, less resistance to frequency selective fading, less inflexibility, high peak to average ratio, and frequency error. In order to overcome these limitations many solutions have been proposed and published in the literatures. Adopting OWDM in DVB-T system is one of them. In this paper it has been shown that the wavelet based DVB-T system outperforms the FFT based DVB-T system provided a suitable wavelet family is chosen. In this paper we investigate a number of wavelet families. We conclude that the Haar wavelet is the best choice for DVB-T system. The Haar wavelet provides the maximum resilience to noise and hence provides a low bit error rate for a given signal-to-noise ratio. It also reduces the amplitude variation of the DVB-T signal.

\section{REFERENCES}

[1] DVB Standards available at www.dvb.org

[2] R. Chang, "Synthesis of a Band-Limited Orthogonal Signals for Multichannel Data Transmission", The Bell Technical Journal, Vol. 45, 1966, pp. 1775 - 1796

[3] Uwe Ladebusch, and Claudi A. Liss, “ Terrestrial DVB (DVB-T): A Broadcast Technology for Stationary Portable and Mobile Use", Proceedings of IEEE, Vol. 94, No. 1, January 2006, pp. 183-193

[4] Peter S., “ DVB: Developing Global Television Standards for Today and Tomorrow”, Technical Seminar at International Telecommunication Union (ITU), 2011

[5] Reimars, U.H., “ DVB-The Family of International Standards for Digital Video Broadcasting”, Proceedings of IEEE, Vol. 4, No. 1,2006, pp. 173-182

[6] R. V. Nee and R. Prasad, OFDM Wireless Multimedia Communications, Norwood, MA: Artech House, 2000.

[7] J. A. C. Bingham, "Multi-carrier modulation for data transmission: An idea whose time has come", IEEE Communications Magazine, vol.28, no. 5, pp.5-14, May 1990.

[8] Man-On Pun, Michele Morelli, and C-C Jay Kuo, "Multi-Carrier Techniques for Broadband Wireless Communications: A Signal Processing Perspective", Imperial College Press, 57 Shelton Street, Covent Garden, London WC2H $9 \mathrm{HE}$.

[9] S. Mallat, "A Theory of multiresolution signal decomposition: the wavelet representation", IEEE Pattern Analysis and Machine Intelligence, Vol. 11, No. 7, pp. 674-693.1989

[10] OFDM Simulation using Matlab available at http://www.ece.gatech.edu/research/ labs/ sarl/ tutorials /OFDM/Tutorial_web.pdf 
Mohammed Tarique; Optimum Wavelet Selection for Orthogonal Wavelet Division Multiplex (OWDM) Based Broadcasting System, Transactions on Networks and Communications, Volume 4 No. 4, August (2016); pp: 1-12

[11] S.L. Linffot and M.K. Ibrahim, “Flexible Modulation for Digital Terrestrial Broadcasting”, IEEE Electronics Letters, Vol. 42, No. 23, November 2006, pp. 1360-1362

[12] Introduction to the wavelet families available at www.mathworks.com

[13] G. Strang, and T. Nguyen, "Wavelets and Filter Banks", Wellesly-Cambridge Press, Massachusetts, USA, 1997

[14] Khaizuran Abdullah and Zahir M. Hussain, "Performance of Fourier-Based and Wavelet-Based OFDM for DVB-T Systems", Proceedings of the Australian Telecommunication Networks and Applications, Christchurch, New Zealand, December 2007, pp. 475-479

[15] Vidushi Dagour, and Prabhat Patel, "Performance of Wavelet Transform based OFDM and Application to DVB system", International Journal of Innovative Research in Science, Engineering and Technology, Vol. 4, No. 8, August 2015, pp. 6760-6767

[16] M.K. Lakshmanan, H. Nikookar, "A Review of Wavelets for Digital Wireless Communication", Wireless Personal Communication, Vol. 37, 2006, pp. 387-420

[17] Volkan Kumbasar, and Oguz Hucur, "Better wavelet packet tree structures for PAPR reduction in WOFDM System”, Digital Signal Processing, Vol. 18, No. 6, 2008, Pages 885-891

[18] Mohammed H. M. Neerma, Nidal S. Kamel, Varon Jeoti," BER Performance Analysis of OFDM System Based on Dual-Tree Complex Wavelet Transform in AWGN Channel", Proceedings of the 8th WSEAS International Conference on Signal Processing, February, 2009, Cambridge, pp. 85-89

[19] Mahesh Kuma Gupta, and S. Tiwari, "Performance evaluation of conventional and wavelet based OFDM system", International Journal of Electronics and Communications, Vol. 67, no. 4, April 2013, pp. 348-354 